\title{
Moral challenges in managed care
}

\author{
L Hattingh, MB ChB, MPhil (Applied Ethics) \\ Leandri Hattingh writes in her personal capacity. Previously affiliated to Stellenbosch University, she now works for Medscheme.
}

\author{
Corresponding author: L Hattingh (leandrihatt@gmail.com)
}

\begin{abstract}
Managed healthcare in South Africa is faced with complex moral challenges, where different stakeholders appeal to different ethics principles to guide decision-making. The traditional bio-medical ethics principles of beneficence, non-maleficence and respect for autonomy are typically emphasised in clinical practice, while third-party funders appeal to the principle of justice to guide the allocation of limited, pooled resources. Healthcare professionals working in managed care are particularly exposed to these conflicts, vis-à-vis incongruence between the ethics guidelines from their professional bodies and the legislation pertaining to managed care. Common understanding of the claims and responsibilities of each of the stakeholders may promote a more coherent, sustainable healthcare system.
\end{abstract}

S Afr J BL 2015;8(2):17-20. DOI:10.7196/SAJBL.431

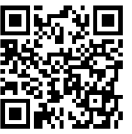

While worldwide debate rages about the ideal healthcare system, managed care is an integral part of the current South African (SA) private healthcare sector, and may become relevant in a future national health insurance structure. Managed healthcare is defined in Regulation 15 of the Medical Schemes Act ${ }^{[1]}$ as:

'clinical and financial risk assessment and management of health care, with a view to facilitating appropriateness and cost-effectiveness of relevant health services within the constraints of what is affordable, through the use of rules-based and clinical management-based programmes.'

In terms of the Act, managed care organisations may be contracted by medical aid schemes to provide the above services. As third-party funders, the influence of medical schemes has historically centred on funding of healthcare services. As managed care programmes move closer towards prescriptive practices to healthcare practitioners, the boundaries of liability between patient, clinician and managed care organisation are in a constant state of flux. An understanding of the ethical responsibilities of each is necessary for protection of all stakeholders in the private healthcare system. These include clinicians, managed care organisations, medical schemes, and patients who also function as beneficiaries of such schemes. This article will focus on the four traditional bio-medical ethics principles as applied to the managed care paradigm, specifically in the context of healthcare professionals employed in managed care, and will explore some of the conflicts as various stakeholders appeal to different principles in guiding their ethical behaviour. Ultimately, the patient remains at the centre of this complex interaction. It falls upon all stakeholders to strive towards a sustainable system which provides reasonable access to healthcare of acceptable quality.

\section{The four biomedical ethics principles}

The principle-based approach to biomedical ethics as suggested prominently by Beauchamp and Childress ${ }^{[2]}$ proposes four main biomedical ethics principles: respect for autonomy, beneficence, non-maleficence and justice. No one principle has prima facie value over another - to decide between conflicting principles requires a process of moral deliberation in a specific practical context. ${ }^{[3]}$ While Petrini and Gainotti ${ }^{[4]}$ suggest that the principle-based approach to biomedical ethics is inadequate to address the questions faced in the public health management and policy environment, it remains the dominant approach to biomedical ethics in SA.

In the managed care context, various stakeholders implicitly appeal to different principles to guide their decision-making. The principles of autonomy, beneficence and non-maleficence are comfortably invoked by medical practitioners in pursuing the best, immediate interests of their individual patients. Managed care organisations and medical schemes, however, are primarily concerned with the principle of justice, as their duties are towards large collections of individuals in the form of medical scheme memberships. They are also concerned about distributive justice across time, as they seek to sustain healthcare funding into the future. These concerns are central to the domains of public health management and policy.

\section{Who gets what? - utilitarianism and its problems}

The sky-rocketing costs of modern healthcare associated with its myriad of technological advances render even standard healthcare unaffordable to most people in their individual capacities. Hence the need for pooled resources such as medical schemes or government funding mechanisms for the majority of healthcare needs. Access to modern healthcare generates not only claims on the individual patient's bank balance, but also on that of society and collectives like medical schemes. ${ }^{[3]}$ In the face of limited resources and increasing healthcare costs, some form of rationing is imperative, and the concept of utility is often appealed to in guiding this rationing towards the greater good for the greater society.

Utilitarianism, as a consequentialist moral theory, holds that moral decision-making should be based on evaluation of the foreseeable consequences of an action, not on the action itself, and that the action which produces the most good consequences 
(roughly translating to promoting the greater good for the greater society), is the right one. Prima facie, this appears to be a reasonable starting point to guide the allocation of shared, limited healthcare resources. One of the main criticisms against utilitarianism, however, is that it clashes with the concepts of rights and fairness. Rachels and Rachels ${ }^{[5]}$ write: 'Utilitarianism is at odds with the idea that people have rights that may not be trampled on merely because one anticipates good results'. Consider the following hypothetical situation to illustrate this point: say there are three people; the only distinguishing factor between them is that two of them need kidney transplants and one has two healthy kidneys (for purposes of this illustration, let us assume that they do not have access to renal dialysis). The utilitarian might argue that the life of the latter must be sacrificed for donation of both her kidneys in order to save the other two; instead of losing two people, now only one will die. The rights to life and health, as well as the ethics principles of autonomy and non-maleficence are all compromised by this approach. That said, a thoroughly considered utilitarian view may take into account the overall (long-term) consequences of forcing the healthy person to donate both kidneys, such as possible social uproar and general disrespect for life that may ensue. This may ultimately sway the utilitarian to decide against such action, purely based on utilitarian principles. ${ }^{[3]}$ Another problem of utilitarianism, however, is the inherent inability to foresee all possible consequences and to predict the future with absolute confidence. ${ }^{[3]}$ The utilitarian may base decisions on insufficient information or incorrect assumptions about the future and this may ultimately lead to failure in achieving the greater good despite the utilitarian's best attempts.

A further important criticism of Jeremy Bentham's (1748 - 1832) concept of utility is that it attempts to measure all moral values on one scale (of happiness), which can easily translate into a single scale of monetary value in the hands of public policymakers such as managed care organisations. ${ }^{[3]}$ Even though this would not be a true interpretation of Bentham's utilitarianism (which would also account for emotional and other consequences of actions), Michael Sande ${ }^{[6]}$ points out that cost-benefit analysis - a common tool used in health economics - has a strong utilitarian underpinning in its attempt to reduce all values to one monetary measure. It would be dangerous to completely ignore concepts such as rights, emotions, sentiments and values attributed to human life and health. These concepts are intricately embedded in the very definitions of health and healthcare. ${ }^{[3]}$

\section{Distributive justice}

To address the problems posed by a purely utilitarian approach to justice in healthcare, an approach such as that of Beauchamp and Childress' $^{\text {'2] }}$ provides a kind of 'safety net' to prevent the vulnerable from facing gross violation of their rights. They describe the principle of justice in the form of a 'general approach' to the questions on social justice and specifically distributive justice in healthcare. This principle is to guide the allocation of health resources and burdens within a society. They propose the recognition of 'an enforceable right to a decent minimum of healthcare within a framework for allocation that incorporates both utilitarian and egalitarian standards'. ${ }^{[2]}$ Their principle of justice draws on the social justice theory of John Rawls, ${ }^{[7]}$ and incorporates his concept of justified unequal distribution, as long as its result is to the benefit of the least advantaged. ${ }^{[3,7]}$

\section{The prescribed minimum benefits: Prescribed minimum beneficence}

The prescribed minimum benefits (PMBs), as described in the regulations to the South African Medical Schemes Act of 1998, ${ }^{[1]}$ recognise the need for provision of a basic minimum level of private healthcare. The regulations enforce the funding of these PMBs for all members of medical aid schemes, in order to alleviate some of the burden of care from the public health sector, and to guarantee access to a minimum level of healthcare coverage regardless of varying contributions from scheme members. The PMB regulations define a list of conditions typically requiring hospital-based care, common chronic conditions, and medical emergencies. The type of care included is further defined by treatment algorithms and prescribed treatments, also published under these regulations. The PMBs (albeit not without shortcomings) can be seen as a practical implementation of Beauchamp and Childress's principle of distributive justice. This enforces a basic level of beneficence towards individual scheme beneficiaries, in order to protect their rights to basic healthcare where conflicts with justice arise. Conflicts may still arise, however, when medical scheme beneficiaries require care which is in excess of the basic minimum. Resource allocation according to the principle of distributive justice still supports rationing at a societal level, and at the coalface of healthcare delivery, this may come into conflict with individual appeals to beneficence, non-maleficence, and respect for autonomy.

\section{Threats to autonomy}

The author predicts that autonomy will increasingly become an important bone of contention in the SA managed care context. Autonomy entails an individual agent exercising freedom of choice, and in a medical context, these choices usually pertain to the agent's body, health, and privacy. According to Beauchamp and Childress, ${ }^{[2]}$ 'liberty (independence from controlling influences) and agency (capacity for intentional action)' are essential conditions for autonomy. The influence of medical schemes and their managed care organisations is primarily focussed on the funding of healthcare. In this context, patients and clinicians have the freedom of choice in the treatments they use or prescribe. Their choice may, however, be limited indirectly by the unavailability of funding. In following international trends, managed care in SA is increasingly moving towards more prescriptive practices to healthcare practitioners, with standardised 'care pathways' and incentives to influence clinician behaviour in a bid to promote cost-effective, quality care. These practices may threaten the autonomy of the patient and clinician.

Another important exercise in autonomy by the medical scheme member occurs when choosing the type of insurance cover to purchase. Members tend to underinsure themselves for various reasons. These include constraints on affordability of premiums, poor understanding of available benefit options and matching these against their own needs, and sometimes, a lack of discipline in providing for future healthcare and financial needs. The Consumer Protection $\mathrm{Act}^{\left[{ }^{[8]}\right.}$ promotes informed decision-making when buying medical insurance products, but the industry still has some work to do to enhance this process. The complexity of the medical schemes legislation (particularly in terms of PMBs) further impedes informed decision-making by scheme members, who may harbour false assurance of insurance cover, which is often subject to complex 
limitations. The unpredictability of some future healthcare needs further complicates this.

\section{Moral complexity in managed care}

To further illustrate the conflicts generated by these philosophical considerations in the practical context, consider the employment of medical professionals by managed care organisations. The regulations to the Medical Schemes Act ${ }^{[1]}$ require that'qualified health care professionals administer the managed healthcare programmes and oversee funding decisions'. While the duties of these professionals are governed by the Medical Schemes Act, they also remain bound by other healthcare laws and ethics rules prescribed by the regulators of their professions. These include the Health Professions Council of South Africa (HPCSA), Nursing Council of South Africa, and the South African Pharmacy Council. The ethics guidelines of these organisations mainly focus on clinical practice, which is indeed relevant to the majority of their members. There are, however, no specific guidelines for the conduct of medical professionals in managed care or other administrative or public health management positions, and some of the existing guidelines and position statements by the HPCSA place these professionals in a precarious position of conflict with the Medical Schemes Act. The following examples demonstrate this:

A policy document on undesirable business practices by the $\mathrm{HPCSA}^{[9]}$ states the following under the section relating to managed healthcare:

'The medical protocols, clinical guidelines and review criteria used by medical schemes and managed care organisations must be developed by doctors according to scientific criteria.

'These guidelines should not be dictated or influenced by managers of $H M O$ (health care management organisations) whose primary objective is cost-saving.'

Another section of the documents states 'The HPCSA holds the view that all healthcare professionals should at all times act in the best interest of the patient and place the clinical needs of the patient paramount ${ }^{[9]}$ If only considering the clinical evidence and ignoring the cost implications, medical professionals employed by managed care organisations would be failing their legal duties in terms of the Medical Schemes Act. Considerations of cost-effectiveness and affordability are as important, as recognised in the Regulations to the Medical Schemes $\mathrm{Act}^{[1]}$, which state: If managed healthcare entails limiting coverage of specific diseases ... such limitations or a restricted list of diseases must be developed on the basis of evidence-based medicine, taking into account considerations of cost-effectiveness and affordability'.

In accordance with these regulations, medical professionals tasked with managed healthcare ought to base their decisions regarding the limitation of healthcare funding on clinical evidence as well as considerations of cost-effectiveness and affordability, and cannot ignore the cost element, as prescribed by the HPCSA guidelines. By ignoring cost, these professionals would also fail in the application of the ethics principle of distributive justice. Pursuing only the best interests of the individual patient ignores the concerns of the broader society, and hampers the fair distribution of commonly-owned resources. ${ }^{[3]}$

This example illustrates the conflict in the managed care environment between the principles of beneficence, along with non- maleficence - underlying the concept of evidence-based medicine, where efficacy and safety are the main concerns - versus distributive justice. It also highlights the inadequacy of the existing professional ethics guidelines for doctors in catering for the complex situation in which medical advisors to medical schemes and managed care organisations function. Of concern is that, while the HPCSA General Ethical Guidelines for the Health Care Professions ${ }^{[10]}$ do not provide for all eventualities by their own admission, they are construed to be '.... fairly comprehensive picture of what it is, in general, that binds any healthcare provider as a professional to his or her patients, as well as to others' and '...that these duties, if not honoured without justification, may constitute the basis for sanctions being imposed on professionals by the Health Professions Council of South Africa! ${ }^{\prime[10]}$ The important consideration of cost in managed healthcare, as legislated by the Regulations to the Medical Schemes Act, ${ }_{1}^{[1]}$ is not accommodated for in the HPCSA guidelines, and action by medical professionals involved in managed care, based on such consideration, may be in conflict with these guidelines.

The Nursing Council and Pharmacy Council's ethical guidelines appear more balanced with due consideration of justice. The Pharmacy Code of Conduct ${ }^{[11]}$ places equal emphasis on duty towards the individual patient and to the public as a whole. The Nursing Code of Conduct ${ }^{[12]}$ is explicitly binding on nursing practitioners in health policy and administration roles, among others. Throughout this document, the importance of larger societal considerations is emphasised. In its detailing of the principle of (social) justice, fairness, equity, and advocacy for the vulnerable are encouraged, also in the context of accessing healthcare resources. This is in contrast with the HPCSA guidelines, ${ }^{[10]}$ where the concept of justice appears to be poorly developed:

'Justice: Health care practitioners should treat all individuals and groups in an impartial, fair and just manner.

'Community: Health care practitioners should strive to contribute to the betterment of society in accordance with their professional abilities and standing in the community.'

In this guideline, practitioners are instructed to 'deal responsibly with scarce healthcare resources', refrain from unnecessary wastage, over servicing and participation in 'improper' financial relationships, while health policy makers are simply instructed to take into account the law, ethics, and human rights. ${ }^{[10]}$ There appears to be a lack of appreciation of the principle of justice in the ethics guidelines for doctors, as compared to those for nurses and pharmacists, while all of these professions play important roles in the managed care and public health environment, where justice is paramount to ethical decision making. It is suggested that a more detailed explanation and emphasis on the concept of distributive justice - one so important in modern healthcare where costs keep rising but resources remain limited, and one that does not come to mind naturally in the western individualist paradigm - is required in the HPCSA guidelines. Doctors - both in managed care and in clinical practice - ought to be guided to not only act in the best interests of the individual patient, without due regard for the broader societal implications, and vice versa. This is applicable not only to considerations of resource limitation, but also to problems such as drug resistance. Irresponsible use of an antibiotic might not harm the individual patient today, but creates 
the opportunity for the development of drug-resistant infection, which has clear implications for society. ${ }^{[3]}$ Distributive justice requires thoughtful deliberation in modern healthcare, both at individual and public levels of the healthcare system.

\section{Recommendations}

All participants in healthcare have to appreciate their roles in a larger system where they have multiple responsibilities toward various other stakeholders. These include patients, healthcare practitioners, funders and managers of healthcare, manufacturers of drugs and equipment, healthcare organisations, society, government and future generations. These responsibilities impose ethical duties on each of these stakeholders, and though they may each have their individual functions and goals, they all need to function in recognition and respect of each other if they are to achieve the over-arching, ultimate goal of any healthcare system: the delivery of quality, universally accessible and sustainable healthcare. ${ }^{[3]}$ Though burdening clinicians for solutions to the problems of health policy and funding would be unfair and unrealistic, it is suggested that a sound appreciation of distributive justice is indispensable in modern medical practice. Doctors and other healthcare practitioners are encouraged to appreciate their broader societal responsibilities and the role they have to play in the just distribution of scarce and unequally distributed healthcare resources. Similarly, managed care organisations, funders and government also need to be cognisant of the claims and aims of all stakeholders in their approach to developing health policy and strategy. ${ }^{[3]}$ As stated by Benatar, Daar and Singer: ${ }^{[13]}$ 'A framework that combines an understanding of global interdependence with enlightened, long-term self-interest has the potential to produce a broad spectrum of beneficial outcomes ...'

One way to protect the autonomy of all stakeholders in the progression towards standardised care pathways in the managed care environment would be the inclusion of each stakeholder in the development of such pathways or guidelines, and the maintenance of exception processes for situations which fall outside the norms. Indeed, the Welsh National Health Services (NHS) mode ${ }^{[14]}$ incorporates reporting on'variance' from the standard as an essential part of a care pathway. This recognises the inherent complexity of medical practice, which frustrates the standardisation of guidelines and care. Unfortunately, exception processes generate additional administration burdens for all involved. The challenge lies in balancing these burdens against the potential gains in quality and efficiency from carefully implemented care pathways. ${ }^{[15]}$

Improved transparency regarding medical aid benefits will support informed decision-making by medical aid members. Brokers for medical insurance products are encouraged to take heed of the importance of well-informed decision-making in this regard. Revision of the PMB legislation, as mandated by the regulations, ${ }^{[1]}$ should, among other things, aim to simplify the legislation and minimise the ambiguity it is currently fraught with. The recent attempts by the Council for Medical Schemes to provide more detailed benefit definitions are encouraging, but will not adequately address certain fundamental flaws in the regulations.

Lastly, there is a lack in ethics guidance for medical professionals working in managed care and health policy management, and the current guidelines by some professional bodies are conflicting and inappropriate for this small but important part of the SA healthcare system, while lacking clear guidance on the application of the principle of justice in modern healthcare. The professional guidelines for healthcare practitioners, and especially those by the HPCSA, may benefit from revision in order to accommodate medical personnel working in managed care, and other public health matters. In its current form, misconceptions about the true definitions of unethical conduct may fuel conflicts between different stakeholders in the healthcare system, particularly between those concerned with individual patient care $\mathrm{v}$. those responsible for public health policy and management. It is hoped that an understanding of the status quo may also help provoke critical appraisal and further development of the current ethics approach to public healthcare and funding in SA.

\section{Conclusion}

The managed care moral landscape is complex. Managed care organisations and medical aid schemes are primarily concerned with the just and sustainable distribution of limited, pooled resources, while clinical practitioners mainly focus on meeting the needs of their individual patients. Patients also tend to be concerned with their individual, immediate needs when faced with threats to their own health, but are forced to contribute to, and utilise, pooled, limited funds from medical schemes for costly medical care. This inevitably brings the ethics principle of justice into conflict with the principles of beneficence, non-maleficence, and respect for autonomy of patient and healthcare professionals. Common understanding of the claims and ethical responsibilities of each of these stakeholders may promote the development of a more coherent, sustainable healthcare system by avoiding undue claims on commonly-owned resources, while ensuring a basic level of acceptable healthcare for all involved.

\section{References}

1. Republic of South Africa. Department of Health (South Africa). Regulations in Terms of the Medical Schemes Act, 1998 (Act No.131 of 1998). Pretoria: Government Gazette 1999.

2. Beauchamp TL, Childress JF. Principles of Biomedical Ethics. 5th ed. New York: Oxford University Press, 2001;58:272.

3. Hattingh L. Business versus health care: Are they compatible? Thesis for MPhil: Applied Ethics. Stellenbosch: Stellenbosch University, 2012:30-92.

4. Petrini C, Gainotti S. A personalist approach to public-health ethics. Bull World Health Organ 2008;86(8):577-656.

5. Rachels J, Rachels S. The Elements of Moral Philosophy. New York: McGraw-Hill, 2010:113.

6. Sandel MJ. Justice. What's the Right Thing to Do? London: Penguin Books Limited, 2010:41.

7. Rawls J. Justice as Fairness (extracts from A Theory of Justice). In: Clayton M, Williams A, eds. Social Justice. Oxford: Blackwell, 2004:60.

8. Republic of South Africa. Consumer Protection Act; Act 68 of 2008. Pretoria: Government Gazette, 29 April 2009.

9. Health Professions Council of South Africa. Policy Document on Undesirable Business Practices. Pretoria: HPCSA, 2005:8-9.

10. Health Professions Council of South Africa. General Ethical Guidelines For The Health Care Professions Booklet 1. Pretoria: HPCSA, May 2008:5.

11. South African Pharmacy Council. The Code of Conduct for Pharmacists and Other Persons Registered in Terms of the Act. The South African Pharmacy Council. http:// www.e2.co.za/emags/CodeofConduct/pageflip.html (accessed 29 Oct 2014).

12. South African Nursing Council. Code of Ethics for Nusing Practitioners in South Africa. Pretoria: South African Nursing Council, May 2013:3-5.

13. Benatar S, Daar AS, Singer PA. Global health ethics: The rationale for mutua caring. In: Benatar S, Brock G, eds. Global Health and Global Heath Ethics. Cambridge: Cambridge University Press, 2011:129-142.

14. Davis N, ed. National Leadership and Innovation Agency for Healthcare. Integrated Care Pathways: A Guide To Good Practice. Llanarhan, Wales: National Leadership and Innovation Agency for Healthcare 2005:7-37.

15. Schrijvers G, van Hoorn A, Huiskes N. The care pathway: Concepts and theories: An introduction. Int J Integr Care 2012;12(Spec Ed Integrated Care Pathways):e192.

16. Republic of South Africa. Medical Schemes Act 131. Pretoria: Government Gazette 1998 